\title{
"DRAGADO DE AGITACION CON BRAZO" EN EL. CANAL DE MARACAIBO - VENEZUELA
}

\author{
Instituto Nacional de Canalizaciones
}

\section{CAPITULO I}

\section{INTRODUCCION}

La cuenca hidrográfica del Lago de Maracaibo situada en la parte Norte de la América del Sur, principalmente en Venezuela, está limitada al Oeste por la Sierra Perijá, al Sur y Sur-Este por el ramal venezolano de la Cordillera de Los Andes, al Este por la línea divisoria entre las aguas que corren al Lago y las que corren al mar Caribe, y por el Norte el Golfo de Venezuela. Su extensión total es de 89756 kilómetros cuadrados de los cuales 73658 están en territorio venezolano y 16098 kilómetros en territorio colombiano; la parte venezolana a su vez se divide en 60555 kilómetros cuadrados de tierra firme y 13103 cubiertos por agua, incluyendo las islas. El aporte de agua dul ce de la cuenca es de 50,57 x $10^{9}$ metros cúbicos anuales aproximadamente, provenientes de numerosos ríos y riachuelos, especialmente am bundantes en la región suroeste.

En el curso de su célebre exploración de la costa Norte de Sur Amé rica, Alonso de Ojeda descubrió, el 24 de agosto de 1499 el Lago de Mă racaibo, llamado Coquivacoa por los naturales, y al cual dió el nombre de San Bartolomé. Alonso Pacheco fundó a Ciudad Rodrigo el 20 de ene ro de 1571, junto al estrecho o Brazo que conecta El Tablazo con el Lăgo. Esta ciudad fue reconstruída en 1574 por Pedro Maldonado quien le cambió el nombre a Nueva Zamora, pero mas tarde se la conoció como Maracaibo y con este último nombre ha llegado hasta nuestros días. Hoy es la población mas grande de la región, es la capital del Estado Zulia, y tiene 457000 habitantes.

Geográficamente podemos dividir al Lago de Maracaibo y sus aguas colindantes en cuatro partes: el Lago propiamente dicho, el Brazo de Ma racaibo, la Bahía de El Tablazo y el Golfo de Venezuela. (Ver Dibujos Nos. 1 y 2). De las tres primeras, la de mayor superficie, es el Lago de Maracaibo el cual tiene $12013 \mathrm{kms}$. cuadrados, con una profundidad media de 25.9 metros (cerca de 85 pies) llegando en algunos sitios hasta los 33.50 metros (110 pies). El $87 \%$ del área del Lago (10 $500 \mathrm{kms.2}$ ) tiene 10 o más metros ( 32.8 pies) de profundidad.

El Brazo de Maracaibo que conecta al Lago con El Tablazo, tiene una longitud de $40 \mathrm{kms}$, un ancho de $6 \mathrm{kms}$. en su extremo Norte y un máximo de $17 \mathrm{kms}$. en su extremo Sur, con un área total de $480 \mathrm{kms}$. 2 , 


\section{COASTAL ENGINEERING}

aproximadamente. A todo lo largo del Brazo corre un canal natural, cuya anchura media es de $1000 \mathrm{mts}$. aproximadamente, con $11.6 \mathrm{mts}$. ( 38 pies)de profundidad mínima y $17.7 \mathrm{mts}$. (58 pies)de profundidad máxima.

La Bahía o estuario de El Tablazo tiene forma trapezoidal y en gene ral es de poca profundidad; limita al Sur con el Brazo de Maracaibo y se comunica con el Golfo de Venezuela por el Canal de la Barra de Maracai bo y las bocas llamadas de Cañonera y Cañonerita. Tiene en el sentido Norte-Sur una longitud de cerca de $24 \mathrm{kms}$, en el Este-Oeste un promedio de 27, y una superficie de $610 \mathrm{kms}$. 2. Es en El Tablazo donde se mezclan las aguas más o menos dulces que vienen del Lago por el Sur, teniendo lugar el movimiento principal de las corrientes a lo largo de un canal que, en su forma natural, serpenteaba por la parte central del estuario y tenía profundidades mínimas de 15 pies.

La costa Sur del Golfo de Venezuela está en constante variación, es pecialmente en la parte correspondiente a las bocas de San Carlos (principal) y Cañonera y Cañonerita (secundarias), debido al encuentro allí del movimiento litoral de arenas cuya dirección general es hacia el Oeste, con las corrientes originadas por la salida de aguas del Lago y las mareas, de rumbo hacia el Norte o Sur. El canal natural de El Tablazo pa saba frente a San Carlos para conectarse con el Golfo después de cruzar la Barra Exterior.

Las zonas de El Tablazo y de la Barra Exterior o Barra de Maracai bo han constituído tradicionalmente el problema básico para la navegāción entre el Lago y el mar libre; solo que la de El Tablazo era algo difí cil pero no peligrosa, mientras que la de la Barra Exterior era tan difí cil como peligrosa. El canal natural que cruzaba ambas zonas tenía en El Tablazo una forma sinuosa relativamente estable en cuanto a posición y profundidad, pero en el cruce de la Barra Exterior, tanto la posición como la profundidad del canal de navegación eran muy variables. El sitio de cruce de la Barra Exterior variaba entre un punto extremo situado a varios $\mathrm{kms}$. al Oeste del Castillo de San Carlos y otro entre San Carlos y Zapara; el canal se deslizaba entre islotes de arena y bancos muy movibles de igual material, que influenciaban tanto su posición como su pro fundidad y atrapaban los barcos que perdían la ruta. Otros factores de inestabilidad lo constituyen la sedimentación de materias en suspensión, las corrientes de densidad, y la floculación en las zonas de contacto del agua dulce con la salada. Esto último tiene lugar solamente en El Tabla zo, especialmente en la zona deltaica alcanzada por las aguas turbias del río Limón, pues las del Lago mismo son perfectamente claras ya que éste último actúa como decantador de sus afluentes.

Las dificultades naturales del acceso al Lago de Maracaibo que aca bamos de describir no tenían mayor esperanza de solución mientras el comercio de la cuenca estuviera limitado a una exportación relativamen 
"DRAGADO DE AGITACION CON BRAZO" EN EL CANAL DE MARACAIBO - VENEZUELA
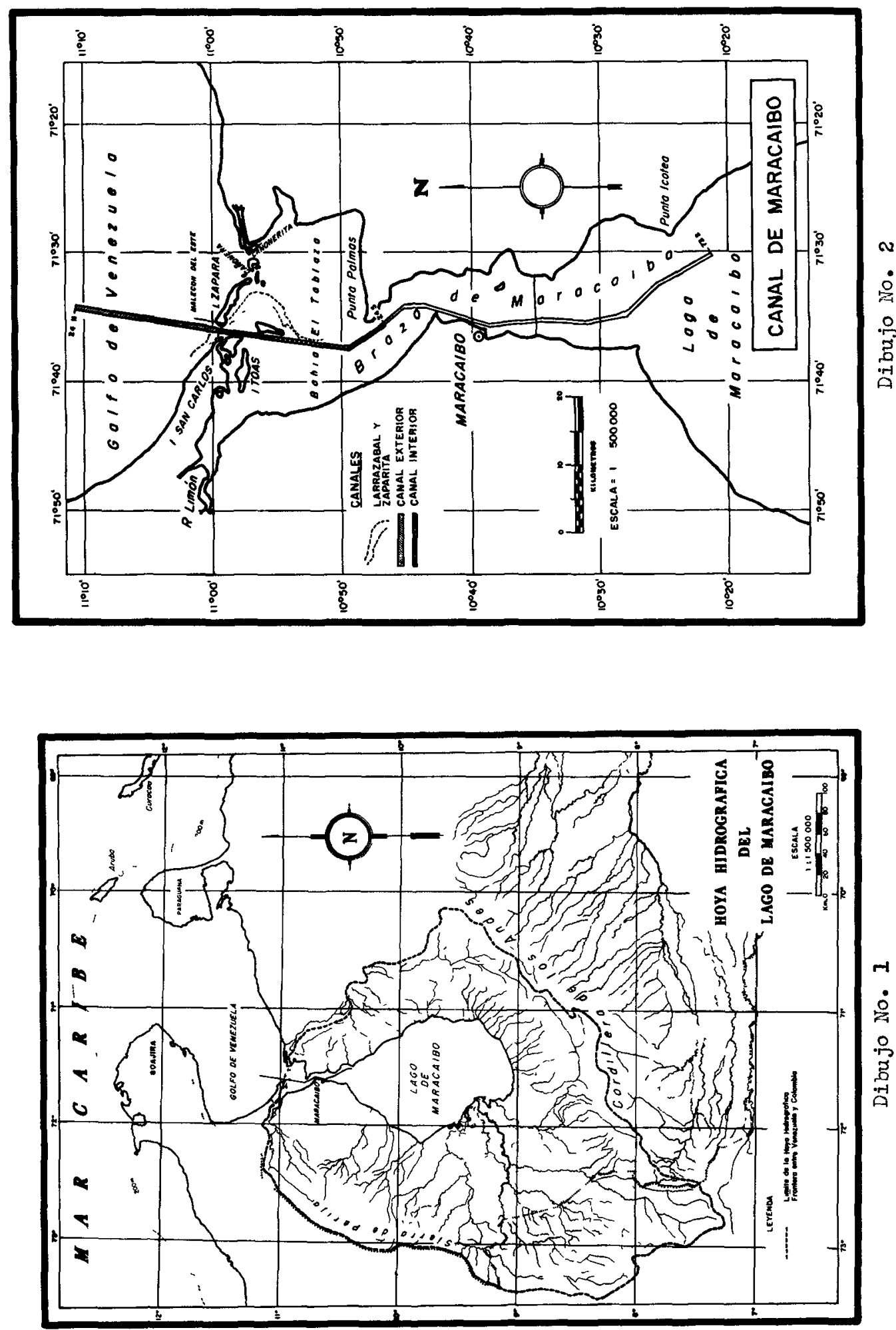
te pequeña de productos agrícolas y pecuarios, y a la importación de va lores similares de bienes de consumo. El descubrimiento del petróleoal requerir el transporte de grandes cantidades de ese producto por la vía marítima, transformó completamente la situación y presentó la jus tificación económica necesaria para la construcción de obras adecuadas de canalización.

\section{CAPIT ULO II}

\section{CONSTRUCCION DEL CANAL}

El proyecto original preparado por el Coronel Robinson del Cuerpo de Ingenieros del Ejército Americano (abreviado U.S.A.C.E.) en 1940 y reformado en 1947, sirvió de base para los trabajos. Dicho proyecto consistía en la construcción de un canal rectilíneo con profundidad de 35 pies referida a la marea media baja (M.M.B.), cuyo rumbo quedó fijado en S. $8^{\circ} 48^{\prime} 40^{\prime \prime} 0$. , tanto para el cruce de la Barra Exterior como para el eje de El Tablazo, con excepción de un pequeño sector al final de este último al cual se le dió un rumbo de S. $29^{\circ} 11^{\prime} 20^{\prime \prime} \mathrm{E}$. , a fin de empatar con el canal natural frente a Punta de Palmas. Este canal quedaba naturalmente dividido en una parte Interior con $182.88 \mathrm{mts}$. (600 pies)de ancho y 22.560 kilómetros de longitud contados hacia el Sur, a partir del punto Cero situado en Zapara, y una parte Exterior con $304.80 \mathrm{mts}$. (1 000 pies) de ancho y $12.450 \mathrm{kms}$. de largo a partir del mismo punto Cero, hacia el Norte. Para la protección de la parte exterior del canal cercana a la costa, se recomendaba la construcción de dos malecones, uno al Este y otro al Oeste, partiendo respectivamente, de las islas de Zapara y San Carlos.

La construcción del Canal Interior se contrató a partir de $0 \mathrm{kms}$. $600 \mathrm{~s}$. hasta $22 \mathrm{kms}$. $560 \mathrm{~S}$., se comenzó el 28 de abril de 1953 y la obra fue inaugurada el 7 de diciembre de 1954. En total se extrajeron - 30655170 metros cúbicos de material en 588 días, con las dragas de tubería Caribbean y Jamaica Bay. En general se puede decir que este dragado, no ofreció mayores problemas.

El día 28 de noviembre de 1954 se iniciaron los trabajos en el Canal Exterior contratados a partir del mismo punto $0 \mathrm{kms}$. $600 \mathrm{~S}$. hasta 12 $\mathrm{kms}$. $450 \mathrm{~N}$., y el 8 de diciembre de 1956 fue inaugurado. En este trabajo tomaron parte en distintas oportunidades tres dragas de tubería, cin co de tolvas y una de brazo, aunque nunca hubo más de tres dragas de tolvas trabajando al mismo ti empo. El volumen removido en los dos años y diez días de trabajo, fue de 20015617 metros cúbicos. El dragado del Canal Exterior fue particularmente difícil debido al tipo de material encontrado cerca de la costa, especialmente entre los Kms. 3 y 6 N. y también por lo agitado del mar, usualmente en los meses de diciembre a marzo. Por este último motivo las dragas de tolvas se vie- 


\section{"DRAGADO DE AGITACION CON BRAZO" EN EL CANAL DE MARACAIBO - VENEZUELA}

ron frecuentemente obligadas a refugiarse en el Canal Interior durante los días de mal tiempo y a fin de realizar trabajo útil dragaban en dicho sector.

Las dragas de tolvas sumaron un total de 46656 horas, 39 minutos de presencia en su trabajo de construcción del Canal Exterior a distribuído de la manera siguiente: el 61,5\% se dedıcó al dragado efectivo, es decir, al bombeo de material, el 9,7\% a mantenimiento, (reparaciones, abastecimiento y otros servicios), el $18,7 \%$ se perdió debido al mal tiempo y el $10,1 \%$ permanecieron inactivas por circunstancias varias.

El Malecón del Este se comenzó a construir el día 19 de febrero de 1954 y se terminó al 14 de junio de 1956. Dicha estructura tiene una longitud de $3250 \mathrm{mts}$. contados desde su punto de arranque en la Costa Norte de la Isla Zapara hasta la línea de 24 pies de profundidad en el Golfo de Venezuela. Su plataforma de coronamiento tiene 7,20 metros de ancho con taludes de $1: 2$ del lado Este y de 1:1,5 por el Oeste. La construcción de piedra suelta, se efectuó con caliza de la isla de Toas, y se emplearon en ella 1100000 toneladas. La construcción del Malecón del Oeste fue aplazada por existir dudas acerca de su conveniencia o necesidad inmediata.

\section{CAPITULO III}

\section{MANTENIMIENTO Y MEJORAS SIN LA DRAGA ZULIA}

El 8 de diciembre de 1956, fecha de conclusión de los trabajos contratados para el Canal Exterior, marca el térmuno oficial del período de construcción; sin embargo, por haberse dragado primero la mitad oriental del canal, se permitió el tráfico de salida para tanqueros tipo $\mathrm{T} 2$ de 18000 toneladas (114000 barriles), con seis meses de anterioridad, es decir desde junıo de 1956. Pero la experiencia adquirida durante el último año de construcción y las tendencias mundiales en cuanto al tama ño de tanqueros, indicaban ya la conveniencia de una serie de mejoras $\bar{a}$ las cuales se dió comienzo inmediatamente después de la inauguración.

En primer lugar se decidió cambiar el nivel de referencia de la ma ra media baja (M.M. B.) que veníamos usando, y con respecto a la cuā teníamos 35 pies de profundidad, por el nivel medio de las mareas mínimas mensuales (M. M. M.) que nos daba solamente $33 \frac{1}{2}$ pies, efectuado este cambio, debíamos profundizar el canal $1 \frac{1}{2}$ pies para mantener los 35 pies proyectados originalmente. En segundo lugar se resolvió que el Canal Interior no era suficientemente seguro con sólo 600 pies de anchura en el fondo, por lo cual convenía elevar esta cifra a 800 pies y finalmente, se llegó a la conclusión de que era necesario aumentar la profun didad de 35 a 45 pies (M. M. M.) debido al incremento en las dimensiones 
de los tanqueros. En este capítulo se presentan los resultados de los esfuerzos del Instituto para llevar a cabo el anterior programa con los equipos disponibles, sin la ayuda de la draga Zulia.

La nueva profundidad de 45 pies (M. M. M.) significó una extensión del dragado hacia el Norte, de 11342 metros hacia el Sur de $14140 \mathrm{me}$ tros, además de tres tramos entre el Brazo y la entrada del Lago, con un total de 19950 metros. El trecho de canal contínuo sometido a man tenimiento tiene ahora, por lo tanto, una longitud total de 60582 metros, y además hay un trecho discontínuo de 40300 metros lo cual arroja un total por supervisar superior a los cien kilómetros (100 882 metros).

El ensanche y gran parte de la profundización del Canal Interior se efectuaron empleando la draga de tubería Jamaica Bay, la cual había quedado inactiva en la zona a la terminación de los trabajos de construc ción. El volumen extraído alcanzó la cantidad de 16293155 de metros cúbicos, y el trabajo se realizó en el período del 21 de junio de 1957 al 21 de febrero de 1960 , en que dicha draga fue retirada por haber comen zado sus actividades la Zulia tres días antes.

En el mantenimiento y profundización del Canal Exterior, estuvieron trabajando cinco dragas de tolvas (sin incluir la Zulia) la última de las cuales fue retirada el 6 de agosto de 1960. En ningún momento trabajaron más de tres de ellas a la vez y cuando el mal tiempo interrumpia su trabajo pasaban a colaborar en el Canal Interior. El volumen dra gado por estasen el período del 5 de diciembre de 1956 al 6 de agosto de 1960, fue de 11464335 metros cúbicos para el Canal Exterior yde 7263862 metros cúbicos para el Canal Interior.

El dragado en la parte Exterior hizo crisis en 1957 pues con las dos dragas de tolvas que tenía bajo contrato, no solamente estaba el Ins tituto incapacitado de efectuar la profundización sino que sus esfuerzos por alcanzar y mantener los 35 pies (M.M. M.), no tenían éxito, y en al gunos sitios se estaba perdiendo profundidad. En esta emergencia $\overline{\mathrm{e}}$ U.S.A.C.E. arrendo al Instituto, por pocos meses, la draga Chester Harding con ayuda de la cual logró recuperar algo de la profundidad per dida.

Basándose en los resultados obtenidos con la draga HAM-302, el Instituto decidió arr endar la nueva draga HAM-303 de iguales características, la cual comenzó a operar el 30 de octubre de 1958. La crisis del equipo de dragado para el mantenimiento del Canal Exterior quedó así aparentemente resuelta empleando las tres dragas de tolvas: HAM302, Sandpiper y HAM-303, pero el ritmo del progreso era tan lento que hubiera sido imposible con ellas solas alcanzar dentro de un tiempo, ni remotamente razonable la profundidad de 45 pies. Por otra parte siendo los costos unitarios obtenidos con el empleo de varias dragas 


\section{"DRAGADO DE AGITACION CON BRAZO" EN EL CANAL DE MARACAIBO - VENEZUELA}

pequeñas, naturalmente superiores a los que se obtendrían con una de gran capacidad, el costo final de la profundización a 45 pies M. M. M. hubiera sido mucho mayor.

Durante el período crítico de 1957, habiamos podido observar el excelente trabajo que estaba realizando en el dragado de Boca Grande, en el Delta del Orinoco, la draga experimental Sealane funcionando con el nuevo sistema de dragado de "agitación" con brazo, que con tan bue nos resultados ya habíamos ensayado por tres (3) semanas en el Canal Exterior de Maracaibo en Noviembre de 1956. Este nuevo tipo de draga, descrito con más detalles en el Capítulo que sigue, parecía ofrecer la única solución satisfactoria de los problemas tanto técnicos como económicos que presentaban el mantenimiento y mejoras del Canal de Ma racaibo y por ello el Instituto resolvió continuar y llevar a feliz térmiño, las negociaciones iniciadas con la National Bulk Carriers (casa matriz de la Seadredge Company, Inc.), para la construcción y arrendamiento de una gran draga de este tipo, la actual Zulia.

\section{CAPITULO IV}

\section{DRAGADO POR AGITACION}

\section{EL DRAGADO POR AGITACION CON DRAGAS DE TOLVAS}

El "Dragado por agitación", efectuado hasta hace poco únicamente con dragas de tolvas, es un método por medio del cual se hace desbordar sobre la superficie del agua toda la mezcla de líquido y sólidos extraídos del fondo, sin buscar o esperar su concentración en las tolvas, a fin de que una parte importante de dicho material sea transportado por las corrientes litorales, fluviales o de mareas que actúen en la zona de trabajo, hasta alcanzar un área de deposición fuera de los límites del Canal. En el libro "The Hopper Dredge" publicado por U.S.A.C.E. en 1954, se detallan el origen y aplicaciones de este método.

El Dibujo No. 3 ha sido preparado por el I. N.C., siguiendo el presentado en la página 308 del libro citado, igualmente basado en la Ley de Stokes para las siguientes condiciones:

$\begin{array}{ll}\text { Gravedad específica de la partícula: } & 2.65 \\ \quad " \quad \text { " del agua: } & 1.00 \\ \text { Aceleración de la gravedad: } & 981 \mathrm{~cm} / \mathrm{seg.} 2 \\ \text { Temperatura del agua: } & 20^{\circ}, 25^{\circ} \text { y } 30^{\circ} \mathrm{C}\end{array}$

Para el uso de este gráfico es necesario determinar el tiempo (T), en minutos, que tarda en caer, en un metro de profundidad, la partícula de mayor tamaño que llega al área de depósito. A este fin se parte de la fórmula siguiente: 


\section{COASTAL ENGINEERING}

$$
\mathrm{T}=0.0167 \times \frac{\mathrm{D}}{\mathrm{p} \times \mathrm{v}} \text { (minutos) }
$$

donde: $\quad D=$ Distancia entre el punto de caída del material agitado y el sitio de depósito; expresada en me tros.

$\mathrm{p}=$ Profundidad del sitio de depósito, en metros.

$\mathrm{v}=$ Velocidad de la corriente, en $\mathrm{mts}$./seg.

Por medio de dicho gráfico se puede estimar el porcentaje del material agitado que sale definitivamente fuera de la zona dragada. Para su empleo se deberán efectuar previamente análisis granulométricos del material, a fin de determinar las proporciones en que se encuentran los tamaños de los granos que lo forman; se necesitará además, estudiar la velocidad y dirección de las corrientes principales, la topografía del fondo y las distancias de las probables trayectorias del material agitado,

Una vez analizada la posibilidad teórica de realizar el dragado por el método de agitación deberán efectuarse exhaustivos ensayos prácticos con la draga destinada al trabajo proyectado, en las condiciones más vam riadas que se espera encontrar en el sitio. Partiendo de estos ensayos y de los volúmenes efectivos a dragar, obtenidos por medio de sondeos, se podrá tomar la decisión acerca del método más adecuado, escogiendo en tre el dragado con tolvas, es decir, cargando y transportando lejos al material excavado, y el método de dragado por agitación en el cual la naturaleza se hace cargo del transporte.

En síntesis, el dragado por agitación con dragas de tolvas, es espe cialmente ventajoso cuando la mayor parte del material extraído del fón do se deposita naturalmente en sitios de donde no puede regresar a $\overline{s u}$ lugar de origen, en un tiempo razonable. Este método no puede emplear se por lo tanto, cuando se opere en aguas tranquilas, o cuando las corrientes predominantes puedan hacer regresar cantidades apreciables de material dragado al Canal o puerto en construcción, o causar su deposición en algún sitio donde exista la posibilidad de dragados futuros.

\section{EL DRAGADO POR AGITACION CON BRAZO}

Casi medio siglo después de iniciado el dragado por agitación se introdujo la variante de lanzar el material extraído lo más lejos posible de la draga, bombeándolo por un conducto o brazo (boom). En esta forma el dragado por agitación se hace aplicable en aguas tranquilas y de mejor rendimiento cuando existen corrientes que arrastren la mayor parte del material dragado fuera de la zona de trabajo. Todos estos aspectos erar de mucho interés en r elación con los canales de Maracaibo y del Orinocc

El estudio del régimen de corrientes, comprendiendo no sólo las de] 
"DRAGADO DE AGITACION CON BRAZO" EN EL CANAL DE MARACAIBO - VENEZUELA

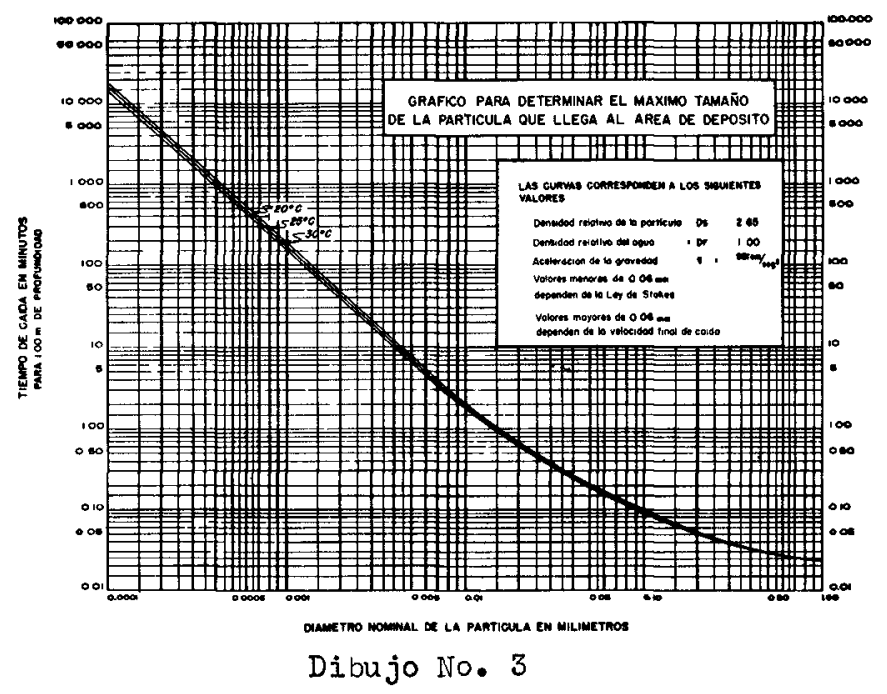

CANAL DE MARACAIBO

OPERAGION DE LA DRAGA "SEALANE" DURANTE EL MES DE MOVEMTER OE 1958

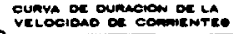
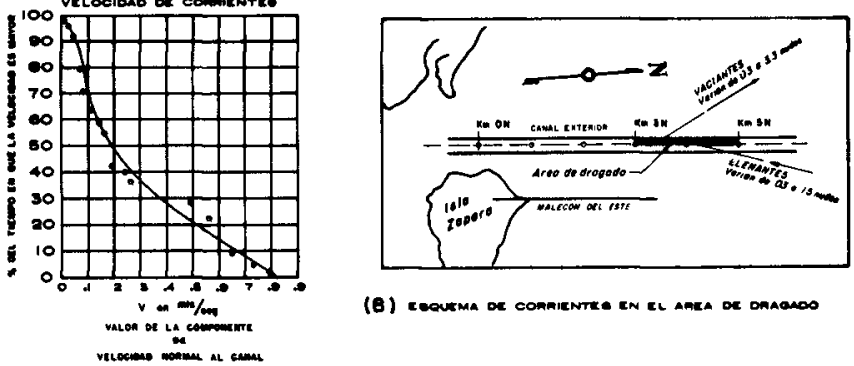

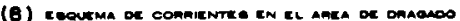

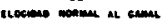

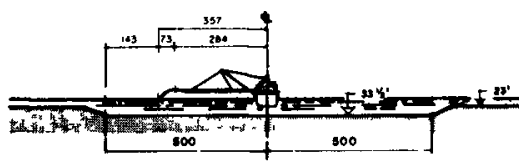

(C)

ormaa - gealane en El canal exterior

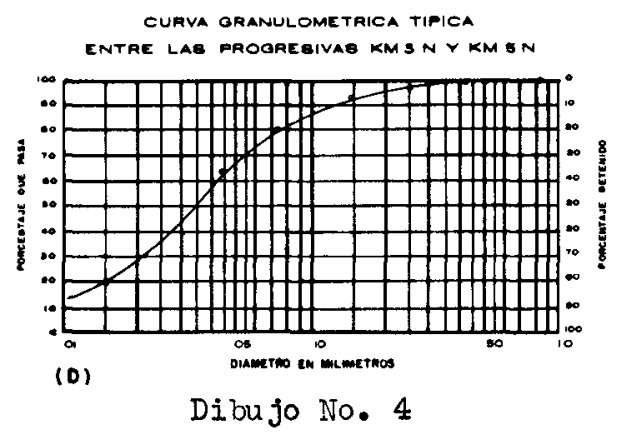




\section{COASTAL ENGINEERING}

río Orinoco sino las originadas de las mareas, y el de la granulometr f́a del material del lecho, basado en numerosas muestras obtenidas por la Orinoco Mining Company, indicaron condiciones favorables al empleo del dragado de agitación con brazo. Como resultado de todas las circuns tancias anteriores, dicha compañía contrató el uso de la draga experimental Sealane.

La Sealane fue construída tomando un tanquero del Tipo T-2 e insta lándole una rastra a cada lado con los correspondientes equipos de bombeo y arreglando la descarga en forma que todo el material fuera expulsado por un brazo formado por dos tubos de 25 pulgadas de diámetro y 76,20 metros de largo medidos a partir del costado del buque. El brazo de descarga sostenido por una trípode, era naturalmente rígido y no ofrecía las ventajas que tendría la draga definitiva cuyo brazo estaría montado en una plataforma giratoria.

El Instituto Nacional de Canalizaciones en conocimiento de las tramitaciones anteriormente mencionadas y con vista a las dificultades que se tenían para terminar la construcción del Canal Exterior (ver Capítu1o II) de la Barra de Maracaibo, obtuvo de la Orinoco Mining Company, permiso para que la Sealane hiciera su primer ensayo en dicho canal an tes de pasar al Orinoco.

La Sealane inició sus trabajos el 7 de noviembre de 1956 y, a fin de utilizar al máximo las corrientes predominantes de la zona las cuales tienen una componente normal al eje del canal, se ordenó limitar el dra gado con brazo a la mitad occidental del Canal Exterior, entre las progresivas $3 \mathrm{Kms} .000 \mathrm{~N}$ y $5 \mathrm{Kms} .000 \mathrm{~N}$ bombeando hacia el Oeste únicamente. De esta manera se buscaba garantizar un mayor volumen de dragado efectivo evitando las contingencias que podían presentarse a consecuencia del bombeo de material hacia el Este, contra la corriente usual, dentro de un canal de 1000 pies de ancho. En la estimación téo rica de la eficiencia de esta operación de dragado que presentaremos más adelante, se podrá apreciar que, efectivamente, esta disposición garantizaba la salida de todo el material bombeado fuera del área del canal.

Por ser éste el primer trabajo de una draga de brazo, creemos in teresante presentar un análisis de las condiciones y características dêl trabajo realizado por la Sealane en el canal de la Barra de Maracaibo.

La distribución del tiempo de trabajo entre las 16:00 horas del 7 de noviembre, cuando comenzó, y las 12:00 del 1 de diciembre cuando terminó, es como sigue: 


\section{"DRAGADO DE AGITACION CON BRAZO" EN EL CANAL DE MARACAIBO - VENEZUELA}

\begin{tabular}{|c|c|}
\hline $\begin{array}{l}\text { Horas de bombeo (dragando): } \\
\text { " en viaje de regreso: } \\
\text { (sin dragar) }\end{array}$ & $\begin{array}{l}316: 55 \\
68: 18 \\
\end{array}$ \\
\hline $\begin{array}{l}\text { Total horas de operación } \\
\text { Demoras por accidentes, re- } \\
\text { paraciones, etc.: }\end{array}$ & $385: 13$ \\
\hline Total de horas empleadas & 572 \\
\hline
\end{tabular}

La producción total estimada a base de las curvas de operación de las bombas fue de $384670 \mathrm{~m} 3$, y de élla se calcular on los siguientes rendimientos:

$\begin{array}{cccc}\text { Producción por hora de bombeo: } & 1214 \mathrm{~m} 3 / \text { hora } \\ \text { " } & \text { " } " \text { "operación: } & 999 & " 1 \\ \text { " } & \text { " } & \text { "presencia: } & 672\end{array}$

Las estimaciones teóricas de que la totalidad del material bombea do por el brazo saldría fuera del canal quedaron confirmadas por sondeos posteriores al dragado. Dichas estimaciones se efectuaron con base a los tres elementos que se describen a continuación:

1) Un estudio aproximado de corrientes en la zona, el cual nos indicó que tanto las originadas por la marea llenante como por la vaciante, mantenĺan unacomponente hacia el oeste, normal al eje del canal según se puede apreciar del Dibujo No. 4-B. Los valores y duración promedio de esta componen te transversal al canal se presentan en forma gráfica mediañ te la curva de duración, del Dibujo No. 4-A, de la misma página.

2) Las diversas posiciones del punto de agitación. En el croquis del Dibujo No. 4-C se indica la ubicación del punto de agitación es decir, el punto de caĺda del chorro de material en la superficie del agua, cuando la draga está en su posición extrema del área de dragado. Este punto varió entre la dis tancia máxima indicada en el croquis, $44 \mathrm{mts}$. (143'), al Este del borde Occidental del Canal, y $109 \mathrm{mts}$. (357') al Oeste del mismo borde. Para la profundidad media del banco se usó 7 mts. (23').

3) La curva granulométrica típica del material dragado por la Sealane, el cual se podría clasificar como un limo fino conteniendo arena muy fina. Esta curva se presenta en el Dibujo No. 4-D. 


\section{COASTAL ENGINEERING}

De la curva de duración de la componente Oeste de la velocidad de corriente, obtenemos $0.10 \mathrm{mts} / \mathrm{seg}$. como el valor sobre el cual estará la velocidad durante el $80 \%$ del tiempo de dragado; dicho valor será usado para obtener las estimaciones de asentamiento del material agitado.

En el cuadro que sigue se calculan los porcentajes de material que salen del canal para varias distancias del punto de agitación a su bordeoccidental mediante la aplicación del gráfico No. 1 mencionado en la pri mera sección de este Capítulo, y del uso de la curva granulométrica del material dragado.

\begin{tabular}{ccccc}
\hline $\begin{array}{c}\text { Distancia al borde } \\
\text { (mts.) }\end{array}$ & $\begin{array}{c}\text { Velocidad corriente } \\
\text { mts/seg. }\end{array}$ & $\begin{array}{c}\mathrm{T} \\
\text { (minutos) }\end{array}$ & $\begin{array}{c}\mathrm{d} \\
\mathrm{m} . \mathrm{m} . \mathrm{m} .\end{array}$ & $\begin{array}{c}\% \text { que sale } \\
\text { del canal }\end{array}$ \\
\hline & & & & \\
44 & 0.10 & 1.136 & 0.14 & 93 \\
24 & 0.10 & 0.572 & 0.22 & 96 \\
12 & 0.10 & 0.286 & 0.35 & 98 \\
\hline
\end{tabular}

De los valores obtenidos en el cuadro anterior para los porcentajes de material que salen del canal se establecía que, aún en las condiciones más desfavorables de posición de la draga, más del 93\% del material bombeado debía considerarse como efectivamente dragado durante más del $80 \%$ del tiempo de operación.

Una vez terminado con éxito este dragado de ensayo en la Barra de Maracaibo, la draga Sealane siguió a la desembocadura del Orinoco para iniciar la construcción del canal de la Barra de Boca Grande. La Sea lane comenzó el dragado a las 10:00 horas del 10 de diciembre de $195 \overline{6}$ y para el 15 de noviembre de 1958 había terminado la construcción de un canal de 48150 metros (26 millas) de longitud, $122 \mathrm{mts}$. (440 pies) de ancho y profundidad mínima de $10.40 \mathrm{mts}$. (34 pies), además de haber realizado otros dragados de menor magnitud aguas arriba del Orinoco.

El trabajo de la Sealane demostró ampliamente la efectividad y eco nomía del dragado de agitación por brazo. Con el empleo de este méto do que alteraba a fondo algunos conceptos sostenidos hasta entonces en materia de dragados, se abrían nuevas perspectivas para dragar y man tener canales como el de Maracaibo y el de la Boca Grande del río Or $\bar{i}-$ noco, cuyos problemas eran antes considerados de muy difícil si no de imposible solución. 


\section{"DRAGADO DE AGITACION CON BRAZO" EN EL CANAL DE MARACAIBO - VENEZUELA}

\section{CAPITULO V}

\section{DESCRIPCION DE LA DRAGA ZULIA}

La urgente necesidad de acelerar la profundización del canal de Maracaibo y de asegurar adecuadamente su mantenimiento, unidas a la imposibilidad de obtener equipos adicionales de dragado, llevaron al Instituto Nacional de Canalizaciones, como se dijo antes, a la conclusión de que era necesario emplear el nuevo sistema de dragado por agi tación con brazo.

Como resultado de esta decisión se contrató con la Seadredge Co. el diseño, construcción y arrendamiento de una draga que pudiera a voluntad, trabajar con brazo o cambiar a tolvas. A esta draga se le dió el nombre "Zulia", en honor del Estado venezolano en el cual se encuentra el Lago de Maracaibo.

La draga Zulıa por la ubicación de sus tolvas en el medio del buque, está clasificada como una draga de tolvas tipo tanquero. En su construcción se tuvo en cuenta toda la experiencia adquirida en el funcionamiento de la draga Essayons del U.S.A.C.E., la cual hasta el momento de la botadura de la Zulia, era la más moderna y efectiva draga de tolvas del mundo. Como draga de tolvas, la draga Zulia desplazó a la Essayons del puesto número uno en el mundo y, como draga de brazo, fue la única en su tipo hasta que se construyó en el mismo as tillero, para trabajar en el Canal del Orinoco, la draga ICOA que es muy similar a élla.

En el cuadro a continuación pueden compararse las características de la draga Zulia con las de la Essayons:

\section{Draga}

Zulia

Entrada en servicio

Año de construcción

Eslora

Manga

Puntal

Largo del brazo (boom):

A partir del eje del barco

" " " costado

Diám. Int. del brazo

Capacidad de tolvas

Calado máximo cargada

$"$ en lastre

\begin{tabular}{|c|c|}
\hline Diciembre 1959 & $\ldots$ \\
\hline 1958 & 1949 \\
\hline $548^{\prime} 6^{\prime \prime}$ & $525^{\prime} 0^{\prime \prime}$ \\
\hline $95^{\prime}$ & $72^{1}$ \\
\hline $40^{\prime} 0^{\prime \prime}$ & $40^{\prime} 5^{\prime \prime}$ \\
\hline $14.5 \mathrm{mts}\left(375^{\prime} 6^{\prime \prime}\right)$ & No tiene \\
\hline $\left.00.0 " 1378^{\prime}\right)$ & $" 1$ \\
\hline $1.45 "\left(57^{\prime}\right)$ & 1111 \\
\hline $\begin{array}{l}6580 \mathrm{~m} 3 \\
26^{\prime} 6^{\prime \prime} \\
16^{\prime}\end{array}$ & $\begin{array}{c}6116 \mathrm{~m} 3 \\
28^{\prime} 0^{\prime \prime \prime}\end{array}$ \\
\hline
\end{tabular}

\section{Draga \\ Essayons}

1949

$525^{\prime} 0^{\prime \prime}$

$72^{1}$ 


\section{COASTAL ENGINEERING}

Profundidad máxima de

dragado

Número y diámetro de tubos de succión

No. y tipo de rastras

No. y tipo de muñoneras

Bombas de dragado:

Número y diámetro

Impulsión de las bombas

Propulsión:

Hélices

Maquinaria

Velocıdad máx. cargada

$60^{\prime}$

\author{
2 laterales de $36^{\prime \prime}$ \\ 2 en fosas de 36 " \\ 4 - California \\ 2 deslizantes \\ 2 fijas
}

4 de $32^{\prime \prime}$

2 turbinas de vapor de $6000 \mathrm{HP} \mathrm{c} / \mathrm{u}$

2 de paso fijo

2 turbinas de vapor con engranaje de re ducción de 6000 $\mathrm{HP} \mathrm{c} / \mathrm{u}$

13 nudos $60^{\prime}$

2 laterales de $36 "$

No tiene

2 - California

2 deslizantes

No tiene

2 de $32^{\prime \prime}$

2 motores eléctricos de $1850 \mathrm{HP} \mathrm{c} / \mathrm{u}$

2 de paso fijo

2 turbinas eléctricas de $4000 \mathrm{HP} \mathrm{c} / \mathrm{u}$

16. 55 nudos

NOTA: La draga ICOA, diseñada especialmente para trabajar en el Orinoco, difiere de la Zulia principalmente en su capacidad de tolvas que es sólo de 2982 metros cúbicos y en el uso de motores diessel en lugar de turbinas a vapor. -

El brazo de la Zulia está constituído por una estructura tubular que soporta, en forma de voladizo, por un lado la tubería aérea dedescarga, y por el otro el contrapeso de 1080 toneladas, necesario para balancear su propio peso y el de la tubería llena de la mezcla dragada.

Mediante un complejo sistema de conexiones, derivaciones, válvulas de paso y válvulas de retención, se combinan las tuberías de descar ga de las cuatro bombas y se puede conducir el flujo del material draga do bien sea a las tolvas, bien al brazo. Dichas bombas tienen una capacidad nominal total de 43150 metros cúbicos por hora para una mezcla con densidad de $1050 \mathrm{gr} / 1$ itro, lo cual equivale a una producción aproximada de $5000 \mathrm{~m} 3 /$ hora de material dragado, considerando una densi dad para el material en sitio, igual a $2000 \mathrm{gr} / 1$ itro.

La tubería de descarga de 32 " de cada bomba empalma en la cubierta principal con una tubería de recolección de $57^{\prime \prime}$ y de ahí sube pa ra conectar con la tubería del brazo aéreo de igual diámetro. Todo el sistema de la tubería de descarga, a partir de su empalme con las bombas está recubierta con goma para evitar la abrasión que produciría en la tubería de acero, la mezcla de agua y sólidos dragados, la cual sale, en forma de impresionante chorro, con velocidad aproximada - 


\section{"DRAGADO DE AGITACION CON BRAZO" EN EL CANAL DE MARACAIBO - VENEZUELA}

de 25 pies por segundo, por la boca de la tubería, situada a 20 metros sobre el nivel del agua.

\section{CAPITULO VI}

\section{ESTUDIOS TEORICOS PARA LA OPERACION DE LA DRAGA ZULIA}

Mientras se construía la Zulia se comenzaron una serie de estudios con objeto de encontrar la manera mas eficiente de emplearla en el Canal de Maracaibo. Dichos estudios han continuado y se continuarán en el futuro, buscando obtener un rendimiento cada vez mayor y más económico.

Fundamentalmente los estudios consistier on en:

a) Establecer las condiciones que determinartan la selección del sistema de dragado que se debía usar para cada caso, es decir, si se dragaba con tolvas o se empleaba el dragado de agitación con brazo, y

b) En caso del dragado con brazo, determinar si se colocaba éste a babor o estribor, en relación con las corrientes predominantes y la granulometría del material dragado.

Para el análisis teórico de estos problemas, se ha preparado el siguiente conjunto de gráficos, en los cuales hacemos uso de las características de sedimentación del material agitado según la composición gra nulométrica:

1) Un gráfico (Dibujo No. 5) que, hecho a base de la fórmula establecida en el Capítulo IV, relaciona los siguientes elementos:

a) La componente normal al canal de la velocidad de corrientes;

b) El cociente que resulta de dividir la distancia (D) del punto de caída del material dragado y la línea de borde del canal, entre la profundidad $(p)$ de dicho borde, $y$

c) El diámetro nominal (d) de la partícula de mayor tama ño que se deposita fuera del canal para una temperatura de $25^{\circ} \mathrm{C}$ y se presentan frente al respectivo valor de " $T$ " en el eje de las abcisas.

2) Sobre un gráfico similar podrían llevarse las curvas granulométricas de los distintos suelos, las cuales nos determinarán los porcen 
tajes de material que, para cada suelo, caen fuera del canal, según el tamaño que determinen la velocidad y la relación $\frac{D}{p} ;$ tal cosa, sin em bargo, no sería práctica por la confusión que significaría el trazado de cien o más curvas en un mismo gráfico. Para evitarlo se han agrupado los suelos según la característica que hemos considerado de mayor importancia en su distribución granulométrica, y la cual se define comoel diámetro nominal de una partícula tal, que el $80 \%$ del material sea más fino que élla. Esta característica, a la cual representamos conel símbolo $\mathrm{D} 80$, ha sido seleccionada con el fin de determinar con ma. yor precisión las cualidades del suelo que se identifica, dentro del ran go de valores comprendidos entre el $60 \%$ y el $100 \%$ de la curva granulo métrica.

Al hacer el agrupamiento de los suelos según el tamaño fíjado del $80 \%$ (D80), se notará que hay variación en la distribución granulométrica para suelos de igual valor de $\mathrm{D} 80$. Se observó que esta variación es de magnitud mayor a medida que aumentan los tamaños del D80 y también a medida que la curva se aleja de los valores de dicho $\mathrm{D} 80$, siendo esto último muy natural por lo explicado en el párrafo anterior. Sin embargo, si se toma en cuenta la precisión dentro de la cual trabajamos, no sólo en lo que se refiere a los métodos de laboratorio utilizados en los análisis de suelos, sino también en lo que se relaciona con lo repre sentativo del muestreo, distribución y medición de la dirección y magñi tud de velocidades de corrientes, estimación de distancias y velocidadreal de caída de las diferentes partículas, podemos considerar que variaciones que se encuentren dentro del margen del $10 \%$ son aceptables para las estimaciones teóricas que pretendemos realizar.

Basados en este criterio, fueron llevados a un gráfico en escalas se milogarítmicas los porcentajes de material más finos que los diámetrōs nominales de $0.010 ; 0.015 ; 0.025 ; 0.05$ y 0.10 , correspondientes a las 72 muestras de suelos tomadas a lo largo del canal, y de acuerdo a sus respectivos valores $\mathrm{D} 80$. Para cada diámetro fuer on trazadas las cur vas promedio obteniéndose la familia de curvas que forman el Dibujo No. 6-A.

Este gráfico nos da, aproximadamente, un promedio de la distribución granulométrica de suelos según su $\mathrm{D} 80$, en forma de porcentajes más finos que las partículas de diámetro " $d$ " que se indican en cada cur va. De él sacamos los valores que utilizamos en el trazado de las curvas promedio granulométricas, correspondientes a los suelos con D80 igual a: 0,$007 ; 0,010 ; 0,015 ; 0,020 ; 0,040 ; 0,070 ; 0,10$ y $0,20,1$ as cuales aparecen superpuestas al gráfico usado para la determinación del diámetro de la partícula de mayor tamaño que sale fuera del canal. (Dibujo No. 5, parte central).

Las curvas granulométricas de aquellos suelos que tengan características muy particulares y que se consideren importantes para el pro- 
"DRAGADO DE AGITACION CON BRAZO" EN EL CANAL DE MARACAIBO - VENEZUELA

DRAGA "ZULIA"

GRAFICO PARA DETERMINAR RENOIMIENTOS
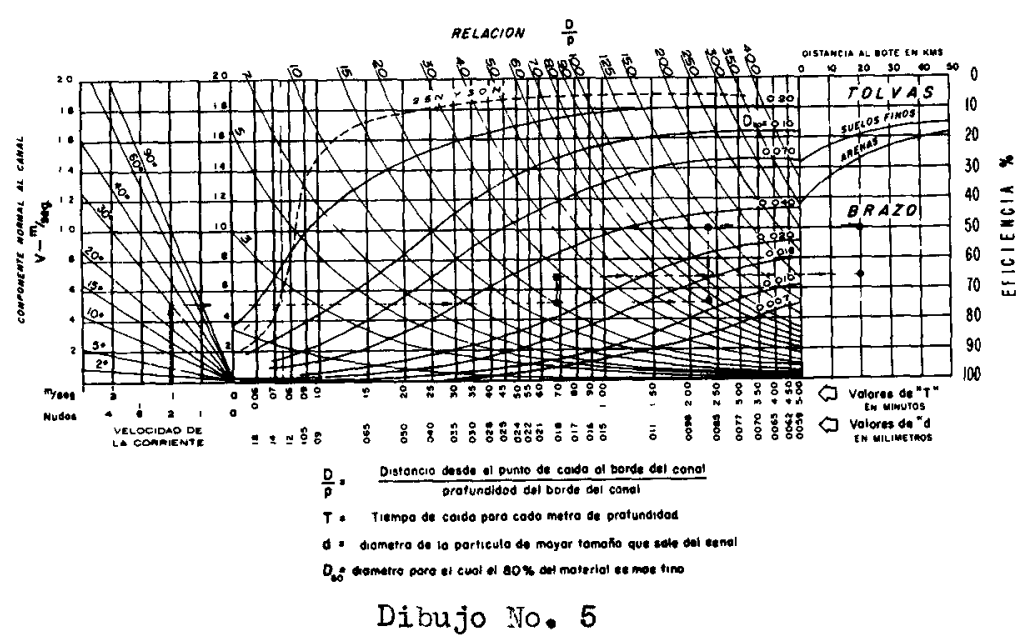

CURVAS PROMEDIO DE DISTRIBUCION GRANULOMETRICA

DE los suelos del FONDO del caNal

de acuerdo al tamaño deL $20 \%\left(D_{B 0}\right)$
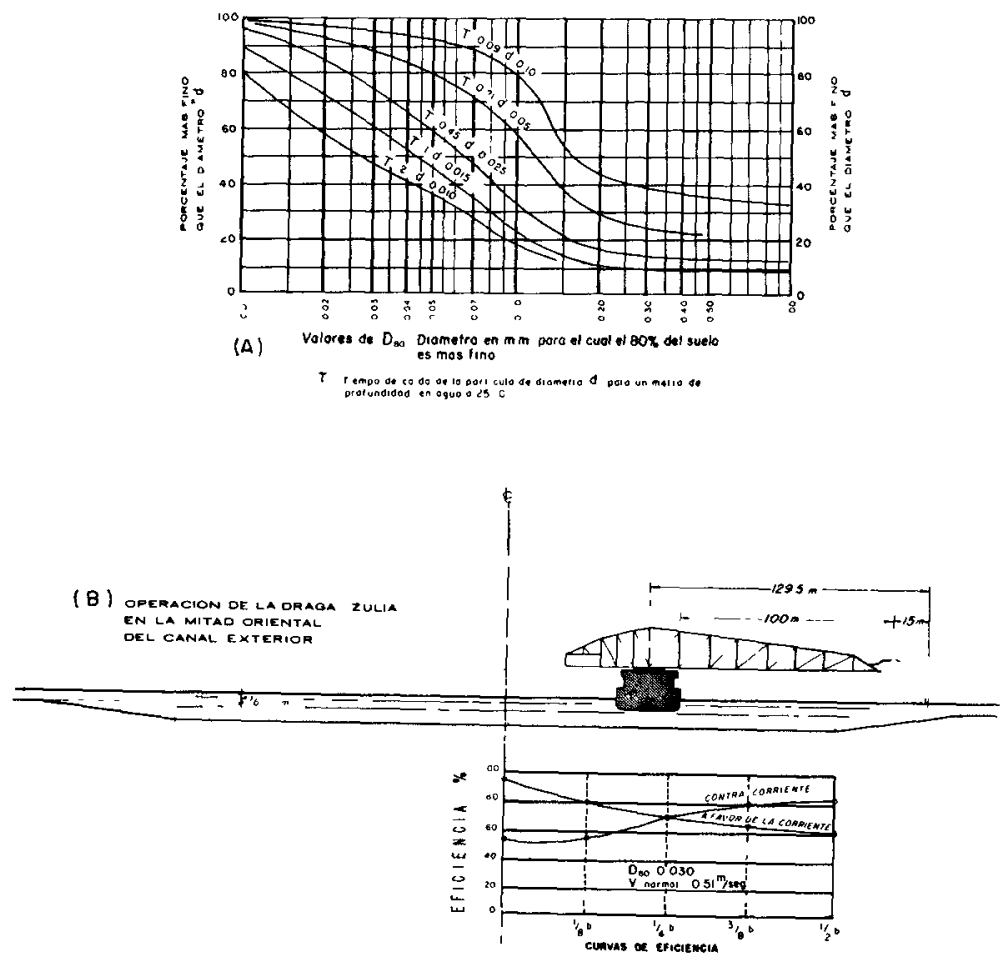

(c)

Dibujo No. 6 


\section{COASTAL ENGINEERING}

ceso de dragado, pueden incluirse especialmente, dentro del gráfico;tal como se ha hecho con la curva que corresponde al suelo entrelas progre sivas $2 \mathrm{kms}$. 500 y $3 \mathrm{kms}$. 000 Norte, debidamente identificada.

3) El Dibujo No. 5 (parte izquierda) nos da la componente normal al canal, de la velocidad de la corriente. Se ha dibujado adyacente al gráfico principal y en él se entra con la magnitud de la corriente en las abcisas, obteniendo el valor de la componente en las ordenadas, según el ángulo de la corriente con el eje del canal.

4) Curva comparativa entre rendimientos del dragado con brazo y del dragado con tolvas para el caso de la Zulia. El objeto de esta com paración es determinar si el volumen efectivo de dragado con brazo és igual o mayor que el dragado con tolvas para un tiempo igual de presencia, escogiéndose el método que resulte mayor.

La comparación se representa mediante la siguiente desigualdad:

$$
\mathrm{Pb} \times \mathrm{Tc} \times \mathrm{E}>\mathrm{V}+
$$

Donde $\mathrm{Pb}=$ Volumen promedio de material bombeado por el brazo

en metros cúbicos por minuto.

Tc- Tiempo de ciclo de la operación con tolvas

$\mathrm{E}$ = Eficiencia del dragado con brazo

$V_{t}=$ Volumen transportado en las tolvas en cada ciclo

Considerando a $\mathrm{Pb}$ y $\mathrm{V}_{\dagger}$ constantes, tendremos como variables a Tc y $E$, las cuales se calculan en los dos números siguientes.

5) El tiempo total del ciclo de operación con tolvas está expresa do por la ecuación:

$$
T c=t l+t e+t v+t d+t r
$$

en la cual:

t1 tiempo de bombeo necesario para llenar las tolvas

te - tiempo de evoluciones

tv = tiempo de viaje al sitio de bote

td = tiempo de descarga de las tolvas

$\operatorname{tr}=$ tiempo de regreso al corte

El tiempo de ciclo se puede considerar constante para un sitio de corte y otro de bote determinados, y para un mismo tipo de suelo draga do.

Los factores que hacen variar el tiempo de ciclo del dragado con 


\section{"DRAGADO DE AGITACION CON BRAZO" EN EL CANAL DE MARACAIBO - VENEZUELA}

tolvas son los siguientes:

a) El tiempo de bombeo necesario para llenar las tolvas ( $t 1$ ) el cual varía de acuerdo con la velocidad de asentamiento de las partículas que forman el suelo, es decir de su granulometría.

Para suelos de grano grueso, el tiempo de carga es menor que pa ra los suelos finos. Esta característica es muy importante y siempre debe ser tomada en cuenta para la programación de los dragados. En el caso de la Zulia, hemos observado que el tiempo de carga varía en tre 160 y 270 minutos según las características de as entamiento del material, pero no se ha tenido todavía la oportunidad de determinar las relaciones que deben existir entre las variaciones granulométricas y las del tiempo de carga. Para tener una idea de cómo nos influiría esto en el dragado, tomaremos las cifras dadas anteriormente para el tiempo de bombeo, como valores extremos entre suelos de grano grueso y suelos de granofino, aptos para ser dragados con tolvas; esta útima observación se debe a que hay suelos cuya granulometría es tan fina que descarta definitivamente el dragado con tolvas por su poco rendimiento.

b) El tiempo de evoluciones (te) es la suma de los tiempos que invierte b draga en suspender el bombeo de material para virar al fin de cada pase. Aunque varía según la longitud del corte y el tiempo de carga, puede considerarse constante ya que estas variaciones son muy pequeñas e influyen poco en el tiempo total del ciclo. Para este tiempo hemos tomado el valor de 32 minutos, que es un promedio obtenido de varias operaciones de la draga Zulia.

c) Los tiempos de viaje para descargar (tv) y de regreso al sitio de corte ( $t r)$ varían con la distancia entre este último y el sitio de bote seleccionado, siempre que se considere uniforme la velocidad de la draga. Por lo regular el sitio de bote permanece fijo, variando estos tiempos solamente según el lugar del corte en el canal. Para nuestro caso, los calcularemos a base de una velocidad de la draga igual a 12 nudos y para los sitios de botes ubicados cerca de las progresivas $11 ; 000$ Norte y $25 ; 000$ Sur del Canal de Maracaibo.

En la práctica los viajes de ida al sitio de bote y regreso al de cor te se aprovechan dragando con brazo durante el trayecto recorrido en el canal, siendo por lo consiguiente los tiempos invertidos mayores que si se efectuara la operación tradicional con tolvas. Sin embargo, para los efectos comparativos que se persiguen, debemos considerar la operación normal es decir, que la draga navega libremente tanto en su viaje al lugar de bote como al regreso de él.

Los tiempos de viaje serán entonces:

$$
\mathrm{tv}=\mathrm{tr}=\frac{\mathrm{L}}{\mathrm{v}}
$$




\section{COASTAL ENGINEERING}

Siendo $\mathrm{v}=12 \mathrm{millas} /$ hor $\mathrm{a}=0.20 \mathrm{millas} / \mathrm{min} .=0.37 \mathrm{~km} / \mathrm{min}$. y es tando $L$ expresado en kilómetros tendremos de acuerdo con la fórmula anterior :

$$
\mathrm{tv}=\mathrm{tr}=\frac{\mathrm{L}}{0.37} \text { (minutos) }=2.70 \times \mathrm{L} \text { (minutos) }
$$

d) El tiempo de descarga de las tolvas (td) es constante e igual a 7 minutos.

e) Sustituyendo los valores obtenidos anteriormente en la relación que nos da el tiempo de ciclo total en minutos, tenemos:

$1 \stackrel{\circ}{\circ}$ Para suelos de grano grueso que llamaremos arenas

$$
\text { tc }=160 \div 32 \div 2 \times 2.70 \mathrm{~L} \div 7=199 \div 5.40 \mathrm{~L}
$$

2) Para suelos de grano fino

$$
\text { tc }=270 \div 32 \div 2 \times 2.70 \mathrm{~L} \div 7=309 \div 5.40 \mathrm{~L}
$$

En ambos caøos I será la distancia del sitio de dragado al sitio de bote, expresado en kilómetros.

6) Como valor de la producción del brazo (Pb) utilizaremos el valor garantizado por las especificaciones de construcción y el contrato de arrendamiento, el cual es de $5070 \mathrm{~m} 3 /$ hora. A esta producción debe mos afectarla de un coeficiente de reducción, debido al tiempo necesario para evoluciones, el cual se estima en 30 minutos cada 6 horas, o sea 5 minutos por hora. La producción efectiva será:

$$
\frac{(60-5)}{60} \times 5070=4647 \mathrm{~m} 3 / \text { hora }=77.45 \mathrm{~m} 3 / \mathrm{minuto}
$$

7) Volviendo ahora a la desigualdad mencionada en el No. 4 anterior para sustituir en ella los valores que acabamos de determinar para Tc y E y consıderando que en la Zulia el volumen de tolvas (Ve) es igual a $6500 \mathrm{~m} 3$ tendremos sustituyendo y despejando E en la desigualdad es tablecida:

$$
77.45 \times \mathrm{Tc} \times \mathrm{E}=6500 \text { de donde } \mathrm{E}=\frac{6500}{77.45 \times \mathrm{TC}}
$$

Sustituyendo a TC por sus respectivos valores y expresando la eficiencia en porcentajes obtendremos:

$$
\text { Para arenas: } E \%=\frac{6500 \times 100}{77.45(199 i 5.40 \mathrm{~L})}=\frac{8393}{199 i 5.40 \mathrm{~L}}
$$




\section{"DRAGADO DE AGITACION CON BRAZO" EN EL CANAL DE MARACAIBO - VENEZUELA}

Para suelos finos: $E \%=\frac{6500 \times 100}{77.45(309+5.40 \mathrm{~L})}=\frac{8393}{309+5.40 \mathrm{~L}}$

Esta desigualdad se representa en el Dibujo No. 5 (parte derecha), que nos relaciona los valores de $\mathrm{E}$ y $\mathrm{L}$, presentado anexo al diagrama que nos da la eficiencia del dragado con brazo, para mayor facilidad.

En este gráfico puede observarse que el tiempo de carga y el de la distancia del sitio de bote, no son los que más influyen en la seleeción del sistema de dragado, siendo más importantes la relación $D / p$ y la componente normal de velocidad.

De la relación establecida entre la eficiencia del dragado con tolvas y brazo, se deduce además que en todo caso, siempre será más económico dragar con brazo dos veces el mismo material (eficiencia $=50 \%$ ) que realizar el dragado con tolvas, con la única condición que el material así dragado sea depositado en un sitio donde no pueda regresar al área de excavación.

Iguales consideraciones pueden establecerse para el caso de que sea necesario dragar tres veces el mismo material, (eficiencia:33.33\%) dependiendo esta vez de la distancia del bote y de la granulometría del material, es decir del tiempo de carga de las tolvas. Asi tenemos que, para suelos finos, será siempre más económico remover el material tres veces para cualquier distancia al sitio de bote. Para arenas, esto será cierto sólo en el caso de que el sitio de bote esté a una distancia mayor de 10 kilómetros del área de dragado.

A partir de lo establecido anteriormente y de las dimensiones del brazo de la draga Zulia, podemos concluir que no será necesario el uso de tolvas para el dragado del Canal de Maracaibo cuyo ancho, $\{1.000$ pies), garantiza una eficiencia del dragado con brazo, superior al $50 \%$ del material bombeado para cualquier condición de corrientes, exceptuando solamente circunstancias especiales como la presencia de obstáculos (por ejemplo las cuatro tor res de enfilación) en cuyo caso sería necesario usar las tolvas.

\section{USO DE LOS GRAFICOS}

Los gráficos que acompañan nos permiten fácilmente analizar para varias condiciones, los rendimientos del dragado con brazo y con tolvas. El siguiente ejemplo servirá para explicar el uso de ellos:

Supongamos que se desee dragar con la Zulia, un tramo de canal de 1.000 pies de ancho, según el croquis y condiciones que se presen$\tan$ en el Dibujo No. 6-B. 


\section{COASTAL ENGINEERING}

Datos:

$$
\begin{aligned}
& \text { Velocidad de la corriente }=2 \text { nudos }=3.70 \mathrm{kms} / \text { hora } \\
& \text { Rumbo " " } "=\mathrm{N} 21^{\circ} \mathrm{W} \\
& " \text { del eje } \quad=N 9^{\circ} \mathrm{E}
\end{aligned}
$$

Distancia media al sitio de bote $=20 \mathrm{kms}$.

La granulometría del suelo señala un D80 $=0.030 \mathrm{~mm}$.

Para estas condiciones de dragado ya hemos establecido anterior mente la mayor economía del dragado con brazo: queda entonces por de terminar la posición que debe darse al brazo según el sitio de la dragā en el canal.

Supongamos que la draga está trabajando en la mitad occidental del Canal o en el eje del mismo. En este caso es evidente que la posición del brazo debe ser a favor de la corriente es decir hacia el Oeste, aún cuando el chorro de material no caiga sobre el borde del Canal.

Pero supongamos ahora que la draga está trabajando en la mitad oriental del canal bajo las mismas condiciones de corrientes, etc., en cuyo caso el material podría descargarse sobre el banco oriental de don de viene la corriente, y consideremos la posición extrema, es decir, que la draga trabaja a lo largu del borde oriental del Canal. Necesitaremos determinar lo siguiente:

1) El material que sedimenta sobre banco oriental del canal

2) El material que sedimenta en el canal

3) El material que alcanza al banco occidental y se sedimenta allí.

Para el primer punto, partimos de los datos:

$$
\mathrm{D}=129.5 \mathrm{~m} . \quad \mathrm{P}=20^{\prime}=6 \mathrm{mts} . \quad \frac{\mathrm{D}}{\mathrm{p}}=21.5
$$

Angulo de la dirección de la corriente con el eje del canal $=21^{\circ} \tau^{\circ}=30^{\circ}$ Intensidad de la corriente $=\mathrm{Vc}=2$ nudos $=1.03 \mathrm{mts} / \mathrm{seg}$.

Entramos en el Dibujo No. 5 con el valor Vc $=1.03 \mathrm{~m} / \mathrm{seg}$. y cor tando la recta correspondiente a los $30^{\circ}$, se determina el valor de la componente normal al canal (v) igual a $0.51 \mathrm{~m} / \mathrm{seg}$. . Seguimos paralelamente a las abcisas hasta encontrar el punto correspondiente a $\mathrm{D} / \mathrm{p}=21$, ! que nos determina la partícula de mayor tamaño que sale del banco oriental cuyo diámetro nominal es de $0.019 \mathrm{~mm}$.. De aquí se localiza en sentido de las ordenadas el punto de corte con la curva granulomé- 


\section{"DRAGADO DE AGITACION CON BRAZO" EN EL \\ CANAL DE MARACAIBO - VENEZUELA}

trica correspondiente a un suelo con $D 80=0.030$, el cual determina una eficiencia del 67\%; es decir, que el 33\% del material bombeado sedimen ta sobre el banco oriental.

Pasando ahora al punto $3^{\circ}$ partimos de los siguientes datos:

$$
D=433.5 p=20^{1}=6 \mathrm{mts} . \quad \frac{D}{p}=72.2
$$

Entramos en el gráfico con los mismosvalores de corriente y llegamos hasta $\mathrm{D} / \mathrm{p}=72.2$, la partícula de mayor tamaño que sedimenta sobre el banco occidental tendrá diámetro nominal igual a $0.0088 \mathrm{~m}$. m. y el punto correspondiente en la familia de curvas granulométricas nos determina una eficiencia del 50\%, para lo cual, el gráfico comparativo de rendimientos entre las operaciones con brazo y con tolvas, nos indica la mayor ventaja del dragado con brazo, para la distancia media de bote establecida $(20 \mathrm{kms}$.).

El punto $22^{\circ}$ se deduce de los dos anteriores, es decir que en el Canal se deposita el $17 \%$ del material bombeado, porcentaje constituído por el material comprendido entre los díametros nominales de $0.0088 \mathrm{~m}$. $\mathrm{m}$. y $0.019 \mathrm{~m} . \mathrm{m}$. .

En síntesis, el análisis teórico de la operación de la draga Zulia en estas condiciones nos da un rendimiento total del $83 \%$. Este valor, com parado con el rendimiento del $60 \%$ que nos da la operación efectuada con el brazo colocado hacia el oeste, es decir en dirección de la corrien te, para el cual $D / p=\frac{174.5}{6} 29$, nos indica que es más efectiva la descār ga contra corriente.

$\mathrm{Si}$,mediante el mismo método, determinamos la eficiencia para las diferentes posiciones que pueda tener la draga en una mitad del canal, ob tendremos las curvas que se presentan en el Dibujo No. 6-C las cuales representan los rendimientos comparativos del dragado con brazo a favor de la corriente y en contra de ella. Para este caso y para estas con diciones se observa que a partir del punto medio de la distancia entre el eje y el borde oriental del canal, es más efectivo el dragado contra corriente.

Refiriéndonos a las curvas de eficiencia del Dibujo 6-C, si llamamos "m" la distancia entre el punto de cambio de 12 orientación del brazo y el eje del canal, podemos establecer que " $m$ " será función de la velocicidad ( $v$ ), de la granulometría del suelo (la cual expresaremos en función de su D80) y de la profundidad de los bancos que limitan el área de dragado $(p)$, o sea:

$$
m=f(v, D 80, p)
$$


COASTAL ENGINEERING
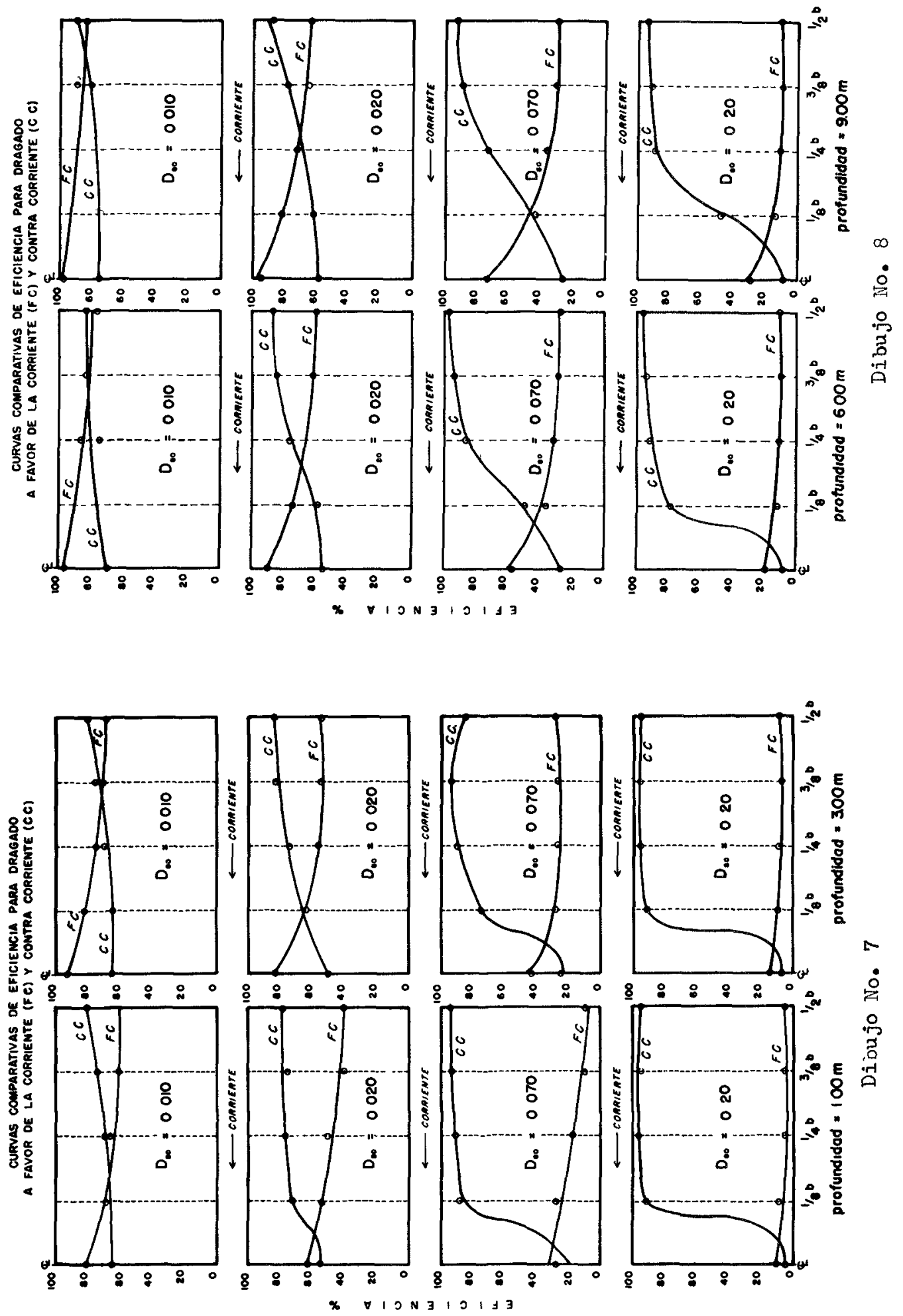


\section{"DRAGADO DE AGITACION CON BRAZO" EN EL CANAL DE MARACAIBO - VENEZUELA}

Si trazamos curvas de eficiencia similares para diferentes valores de los parámetros v, D80 y p observaremos, de manera general lo siguiente:

1) Para suelos finos tales como limos y arcillas, y para los va lores más frecuentes de la velocidad de corriente, la variación de " $m$ " es mayor que para arenas, sin embargo, la diferencia que existe entre la eficiencia para una y otra condición, no pasa del $15 \%$ durante la ma. yor parte del tiempo.

2) Para suelos arenosos con $D 80$ mayor de $0.06 \mathrm{~mm}$. la distancia " $m$ " al punto de cambiar es menor que $1 / 8 \mathrm{del}$ ancho del canal, y la eficiencia, dragando contra la corriente a partir de ese punto, es notablemente mayor que operando a favor de ella.

Esto se puede apreciar muy bien de las curvas de eficiencia obteni das para diferentes profundidades y para suelos con D80 igual a $0.01 \overline{0 ;}$ $0.020 ; 0.070$ y 0.20 milímetros (ver Dibujos Nos. 7 y 8), para los cuales se usa el valor promedio de la componente normal al canal: 0.20 $\mathrm{m} / \mathrm{seg}$., obtenido durante los estudios de corriente del canal exterior de Maracaibo. (Veánse Capítulo IV y Dibujo No. 4-A).

De estos resultados se establece que, en caso de frecuente tráfico por el canal, podría ser ventajoso operar con el brazo hacia el banco más próximo a la draga, independientemente del sentido de la corriente, ya que aún en los casos en que teóricamente exista un ligero aumento de la eficiencia cuando se descarga en sentido contrario, la diferencia podría ser tan pequeña que no compensase el tiempo perdido en maniobras de giración del brazo cada vez que haya que dar paso a los buques que transitan por el canal, ni sea suficiente para justificar el riesgo, por pequeño que este sea, que dicha maniobra conlleva.

Es evidente que análisis teóricos de esta clase, sólo permiten esta blecer normas generales para la operación de la draga, que estarán más o menos ajustadas a la realidad según la precisión con la cual sean determinados los siguientes parámetros básicos: a) el régimen de corrientes y b) las características y distribución granulométrica del material por dragar.

Las dificultades prácticas existentes para una determinación precisa de estos parámetros a todo lo largo del canal y para cualquier tiempo de operación, obligan a una verificación constante de los rendimientos efectivos del dragado mediante anális is comparativos de operación de la draga, basados en frecuentes sondeos del canal; siendo estos análisis los que en definitiva nos permitirán establecer las mejores normas de trabajo. 


\title{
COASTAL ENGINEERING
}

\author{
CAPITULO VII
}

\section{RESULTADOS OBTENIDOS CON LA DRAGA ZULIA}

A continuación presentamos un resumen de los resultados de la ope ración de la Zulia en el Canal de Maracaibo desde el inicio de sus tra jos el 17 de febrero de 1960 hasta el 17 de julio de 1962.

Durante este período de dos años y cinco meses la draga Zulia trabajó a todo lo largo del Canal de Maracaibo desde su extremo norte has ta el extremo sur. La distribución de volúmenes extraídos se señala en los cuadros Nos. 9, 10, 11, 12, 13 y 14 .

Puede notarse que en este tiempo la Zulia ha bombeado a través del brazo la cantidad de $104663000 \mathrm{~m} 3$ de material y dragado por medio de tolvas $2801000 \mathrm{~m} 3$ lo cual arroja un gran total de $107464000 \mathrm{~m} 3$. Por otra parte los volúmenes de profundización y de sedimentación, calculados mediante planos de sondeos, indican que el total de material extraído por la Zulia por el método de agitación con brazo, representa un volumer efectivo de dragado igual a $70462000 \mathrm{~m} 3$. , lo cual nos da una eficiencia promedio para dicho método, abarcando todo el canal dentro del período considerado, igual al $67,32 \%$.

Es necesario admitir sin embargo, que existen dificultades para determinar, con la exactitud deseable, los rendimientos efectivos obtenidos en cada kilómetro. Estas dificultades se pueden resumir de la siguiente manera:

1) La máxima precisión que puede obtenerse en el sondeo es de $0.30 \mathrm{mts}$. (un pie) lo cual representa una posibilidad de error en las es timaciones volumetricas, para un canal de $305 \mathrm{mts}$. de ancho $\left(1000^{\prime}\right)$, que puede llegar a ser igual a $50000 \mathrm{~m} 3 /$ por kilómetro. Este error pue de disminuir y aún ser despreciable, en aquellos casos en que el espesō de la capa dragada sea relativamente grande (mayor de cinco pies); sin embargo para volúmenes de dragado relativamente pequeños la in vitable imprecisión instrumental de los sondeos influye en forma importante.

2) La rata de bombeo y el porcentaje de sólidos de la mezcla varían constantemente durante todo el proceso de dragado y los métodos usados para su determinación son relativamente imprecisos. Las cifras que se usan, por lo regular, son promedios estadísticos, válidos solamente para un gran número de valores.

3) La distribución del tiempo de operación por unidad de longitud de canal, sufre también de algunas imprerisiones, por causa de su distribución proporcional en el intervalo comprendido entre las progresivas 
"DRAGADO DE AGITACION CON BRAZO" EN EL CANAL DE MARACAIBO - VENEZUELA

Cuadro No. 9

\begin{tabular}{|c|c|c|c|c|c|c|c|c|c|c|c|c|c|c|}
\hline \multicolumn{15}{|c|}{ CAPACTERISTICAS DEL CARAGADO OE LA RULIA EN EL TANAL OE MARACABOO } \\
\hline & \multicolumn{5}{|c|}{ GEANULOMETFIA } & & \multirow{2}{*}{ 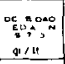 } & \multirow{2}{*}{$\therefore \therefore$} & \multirow{2}{*}{ 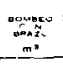 } & \multirow{2}{*}{$\begin{array}{cc}\because & \cdots \\
m^{2} & 0\end{array}$} & \multirow{2}{*}{$\mid$} & \multirow{2}{*}{ 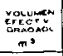 } & \multirow{2}{*}{ 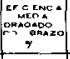 } & \multirow{2}{*}{ 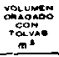 } \\
\hline & $D_{n \infty}^{\infty}$ & $\operatorname{lom}_{1 \rightarrow \infty}$ & & 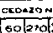 & & & & & & & & & & \\
\hline №nII & & & & & & & & & & & & & & \\
\hline 0.1 & 3 & 100 & of 74 & 19, & 2 & 210 & 1925 & 140 & 2009000 & 533000 & 002000 & 1260000 & 610 & 67000 \\
\hline 12 & 13 & 200 & 4 & 401. & $\cdot$ & 240 & 1935 & $13:$ & 2079000 & 733500 & nos 000 & 1274000 & 613 & 67000 \\
\hline 23 & 0 is & ood & 100 & $9+11$ & ? & 320 & 1950 & $\because 0$ & 3180000 & 997000 & 1192000 & 2169000 & be. : & \\
\hline 34 & 0002 & $\operatorname{cod}$ & 100100 & $\left|x_{6} b_{3}\right|$ & . & 200 & 1945 & 110 & 40000 & 007000 & 2460000 & 3467000 & 703 & \\
\hline 4 & 0077 & & 1001900 & 97 & 14 & 730 & 1940 & $3 \mathrm{~s}$ & 5400000 & 802000 & 2912000 & 3794000 & 702 & \\
\hline 36 & 0.035 & & 100100 & 100 & 60 & 790 & 1925 & is 5 & 6069000 & 949000 & 3311000 & - 260000 & 702 & \\
\hline 67 & 10028 & 100 & log 100 & 100 & 62 & $\Delta 03$ & 1900 & 170 & 50060000 & 1012000 & I 2707000 & $\mid 3700000$ & 60 & \\
\hline 74 & 0026 & 100 & $100 / 100$ & 1009 & $\leftrightarrow$ & 900 & 1050 & 190 & 4202000 & 910000 & 19310000 & 2921000 & 693 & \\
\hline$\because 9$ & 0028 & & $100(100$ & 49,92 & 43 & 980 & 1920 & 22 & 3131000 & 034000 & | 3909000 | & 2213000 & 707 & \\
\hline 910 & 0030 & $\log$ & $100 / 100$ & $100 \mid 93$ & 43 & 900 & $m$ & 245 & $1963000^{\circ}$ & 012000 & 696000 & 1500000 & 760 & \\
\hline 1011 & 0060 & $\log$ & 1000100 & $m$ & 32 & 1000 & 1750 & 265 & 190000 & $1+121000$ & 200000 & $2+00000$ & 140 & \\
\hline 1112 & 0030 & $\log 1$ & $100 \mid 100$ & 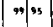 & ${ }^{30}$ & 1040 & 133 & 240 & 1531000 & $\begin{array}{lll}2001 & 000\end{array}$ & 31000 & 1059000 & 692 & \\
\hline 1213 & 0.025 & 100 & 1900 & 1009 & & 1040 & 1725 & 205 & 1531000 & $1003000 \mid$ & 31000 & 1034000 & the . & \\
\hline 1914 & & & & & & 1070 & 1725 & 290 & 15310000 & 1423000 & 60000 & 1183000 & 3 & \\
\hline 1413 & & & & & & $11 \Delta 0$ & 1733 & 190 & 1531000 & 2056000 & • & 2117000 & 30 & \\
\hline 1316 & & & & & & 1130 & 1725 & 310 & $\mid \begin{array}{ll}1332000 \\
\end{array}$ & $9 \cos$ & s 000 & 1007,000 & 103 & \\
\hline 1613 & & & & & & 1130 & 123 & 330 & 12220000 & 890000 & 10000 & 803000 & 3 & \\
\hline 1718 & & & & & & 1260 & 1 ino & 14 & 1098000 & 751000 & 20000 & 717006 & 3 & \\
\hline
\end{tabular}

Cuadro No. 10

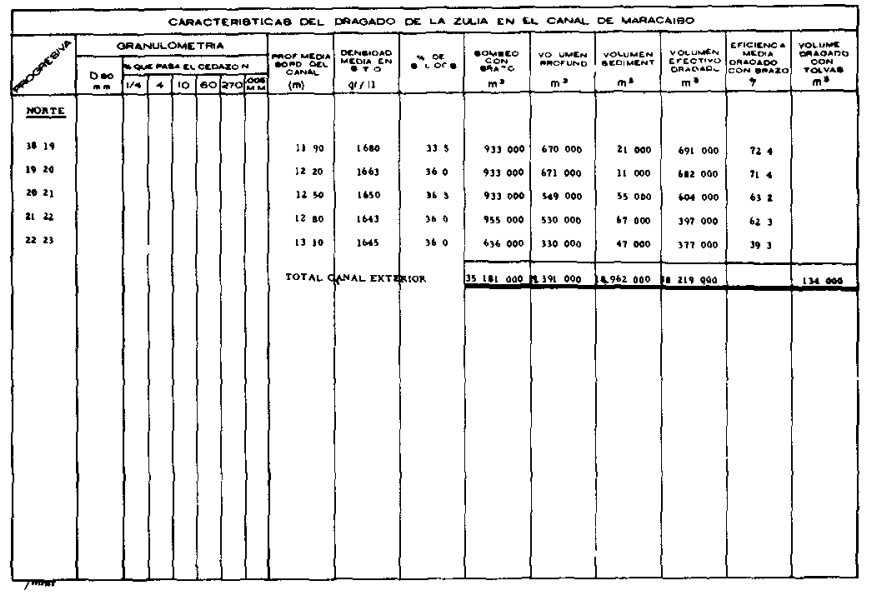

Cuadro No. 11

\begin{tabular}{|c|c|c|c|c|c|c|c|c|c|c|c|c|c|c|}
\hline \multicolumn{15}{|c|}{ 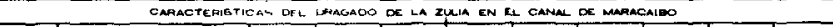 } \\
\hline & & ORANA & 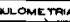 & & & & & & & & & & & \\
\hline & $\begin{array}{c}0.0 \\
m m\end{array}$ & & $\frac{\pi}{4710} 100$ & & & 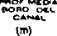 & 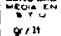 & 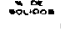 & 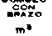 & mats & rgement & 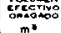 & 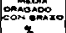 & 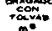 \\
\hline$\because$ & 0.6 & 100 & $\log 100$ & 27 & & 105 & 1910 & 150 & 160000 & 462000 & .92000 & 100000 & $\infty 0$ & ins 000 \\
\hline 12 & 0000 & $1 \infty$ & $\log 1000$ & .7 & & 457 & Lะ70 & 170 & 137600 & 693000 & 63900 & 823000 & mo & $4+3000$ \\
\hline 23 & 011 & 100 & 2001000 & 3 & & 150 & Laso & 105 & 101000 & 299000 & 650000 & 192000 & 629 & 117000 \\
\hline 34 & .15 & 100 & $\log \log 93$ & 20 & & 363 & 1940 & $21 \mathrm{~s}$ & 140100 & 205000 & 621000 & 459000 & 6) 3 & 40000 \\
\hline a & 019 & 100 & 100 & 12 &. & 365 & 1780 & 233 & 165100 & 614000 & 6990000 & 06000 & 326 & itt 000 \\
\hline 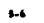 & 0.10 & 100 & $\operatorname{lon} 100 x$ & 45 & 23 & 2,13 & 1778 & 260 & 171400 & 631000 & 692000 & 1018000 & 6) 2 & 234000 \\
\hline $6 \rightarrow$ & 020 & 100 & 10010095 & 19 & . & 200 & 1730 & 230 & 16300 & 430000 & 765000 & 1004000 & 600 & 2340000 \\
\hline r. & 0 ous & 100 & of $\log n$ & 10 & . & 120 & 1740 & 273 & $1 m+\infty$ & ace 000 & 302000 & 935000 & br 1 & $\because 000$ \\
\hline$m$ & 010 & 100 & $\log 1009$ & so & 36 & 120 & 1750 & 240 & 131200 & 322000 & 392000 & ass noos & 1 & 39000 \\
\hline, 10 & $\ldots$ & 100 & 1001000 & 75 & 29 & 275 & 2190 & 203 & 120000 & 5400000 & 300000 & 101000 & 673 & 39000 \\
\hline 16-31 & 00 & 300 & $\cot 100491$ & $\omega$ & 29 & 300 & 1760 & 290 & 107000 & 1230000 & 300000 & 723000 & 67. & \\
\hline $33-12$ & 0 & 300 & $300 \log 97$ & 100 & 20 & 363 & 1770 & 293 & 10040 & . 120000 & 230000 & 720000 & 696 & \\
\hline 1213 & 0.9 & 100 & $\log 1009$ & 56 & 20 & 395 & אדרו & 300 & 109400 & 160000 & 250900 & 710000 & 49 & \\
\hline 1314 & 00 & 100 & $1004 \log 97$ & $\infty$ & 24 & 157 & 1700 & 300 & 10940 & 3050000 & 240000 & 755000 & 60 & \\
\hline 34.13 & 0,035 & 100 & 100 lod 97 & 19 & & 320 & נרו1 & 293 & 109400 & 490000 & 224000 & תות 000 & 656 & \\
\hline 3516 & 010 & 100 & $\log \log 9$ & 53 & 13 & 365 & 1799 & 293 & 10 ont 10 & 162000 & 1600000 & 621000 & " 4 & \\
\hline 1617 & 0 is & 300 & $\log 100=7$ & 38 & 10 & 393 & 1790 & 293 & 100200 & 129000 & 328000 & 637000 & 609 & \\
\hline 1714 & 0 & 100 & 1of iof 99 & 30 & 25 & 485 & (17) & 290 & 1 ote 00 & $\Delta 80000$ & 297000 & mod & 7,3 & \\
\hline 10 & 009 & 100 & 100 100 96 & $\$ 6:$ & 22 & 157 & 1740 & 290 & 100420 & 3600000 & .06000 & 766000 & 10. & \\
\hline 1920 & $0 *$ & 100 & $\log (00 x)$ & $m$ & 20 & $\infty \infty$ & 1773 & 240 & 1002 & .2270000 & 100000 & 17000 & 570 & \\
\hline
\end{tabular}


COASTAL ENGINEERING

Cuadro No. 12

\begin{tabular}{|c|c|c|c|c|c|c|c|c|c|c|c|c|c|c|}
\hline \multicolumn{15}{|c|}{ 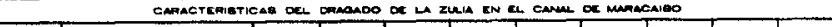 } \\
\hline & \multicolumn{5}{|c|}{ 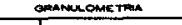 } & & \multirow{2}{*}{ 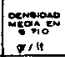 } & \multirow[b]{2}{*}{ 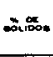 } & \multirow[b]{2}{*}{ 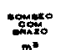 } & \multirow[b]{2}{*}{ 我 } & \multirow[b]{2}{*}{$\begin{array}{c}\text { TimeneN } \\
m^{*}\end{array}$} & \multirow{2}{*}{ 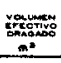 } & \multirow{2}{*}{ wats } & \multirow{2}{*}{ 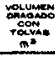 } \\
\hline & $\cdots$ & The mat & 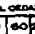 & now & & & & & & & & & & \\
\hline करो & 0.07 & [100/100/100 & $n$ & $n$ & $\infty$ & 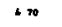 & ins & ans & 1,002000 & $4 m \infty$ & 300000 & 677000 & 62.6 & \\
\hline 212 & ow & 1000 $1000 \mid 100$ & 15 & & 23 & 190 & mo & \#० & 1002,000 & 532.000 & 227000 & 399000 & 701 & \\
\hline 22-s & 007 & $1000|100| 100$ & & "1 & 24. & 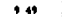 & 1765 & 270 & 2323000 & $=000000$ & 765000 & 2319000 & (2) & \\
\hline 2) & & & & & & 100 & 1760 & iso & : 4020000 & sse.000 & 311000 & $m 000$ & se. 7 & \\
\hline 2425 & & & & & & 1010 & 1755 & 25 5 & in 220000 & 102.000 & 972. 006 & I 124.000 & 6s1 & \\
\hline 2584 & & & & & & 11 so & 1750 & 230 & 1155000 & 1600000 & 1500000 & 301000 & soo & \\
\hline $4 n$ & & & & & & 1010 & 1740 & 235 & I Iss abo & 40000 & S4, 4000 & and 000 & $\infty 0$ & \\
\hline $27-20$ & & & & & & 1130 & 1735 & 200 & $M \infty$ & 6r oose & 306000 & 319000 & 990 & \\
\hline 3029 & & & & & & 1130 & 130 & 750 & 550000 & 33000 & 277000 & 330000 & 600 & \\
\hline 3430 & & & & & & 1230 & 1725 & 255 & 2060000 & is 0000 & 252000 & 106000 & 675 & \\
\hline 3031 & & & & & & 1400 & 1720 & 265 & 502000 & 133000 & 115000 & 300000 & 61, & \\
\hline 33 & & & & & & 1250 & 1715 & 270 & 266000 & 47000 & 119000 & 166000 & 675 & \\
\hline 323 & & & & & & 1220 & 17005 & 275 & 2060000 & 132000 & 14000 & 160000 & 6 & \\
\hline 34 & & & & & & 1220 & 1900 & 280 & 260000 & 1000000 & 16096 & 140000 & 67, & \\
\hline 3635 & & & & & & $11 \infty$ & 1700 & xa 5 & 2700000 & 70000 & $\$ 000$ & 1660000 & 615 & \\
\hline so st & & & & & & $1 \$ 10$ & 1720 & ze s & $14 \times$ & $\infty 000$ & & \$000 & 62 & \\
\hline 51.52 & & & & & & 1240 & $1+00$ & $34 \mathrm{~s}$ & 1400000 & $* 000$ & & 20000 & 671 & \\
\hline$*-n$ & & & & & & 12.50 & 1750 & 300 & 324000 & 220000 & & 220000 & 61 & \\
\hline 3-3-54 & & & & & & 1250 & 1773 & 300 & & & & & & \\
\hline s" & & & & & & 132 & nins & "' & 20000 & tree ono & & 2000000 & 10 & \\
\hline
\end{tabular}

Cuadro No. 13

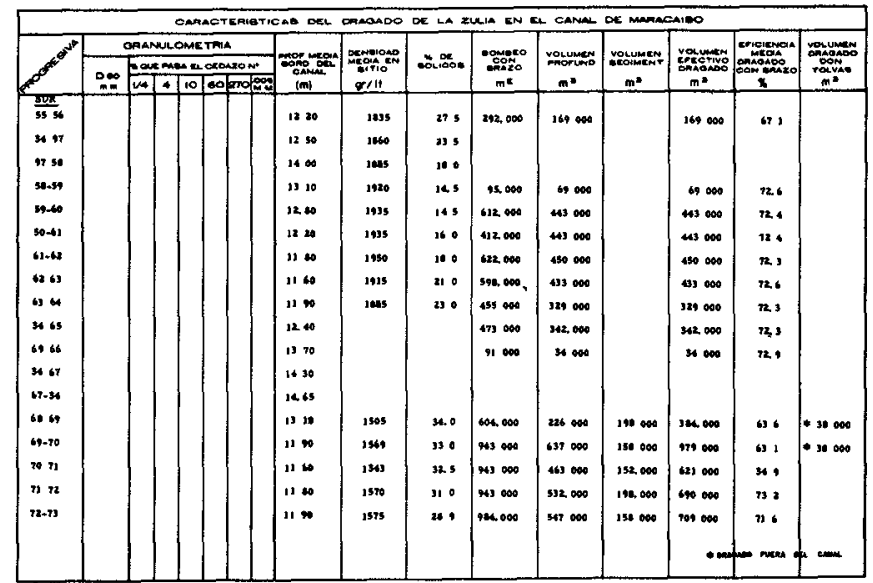

Cuadro No. 14

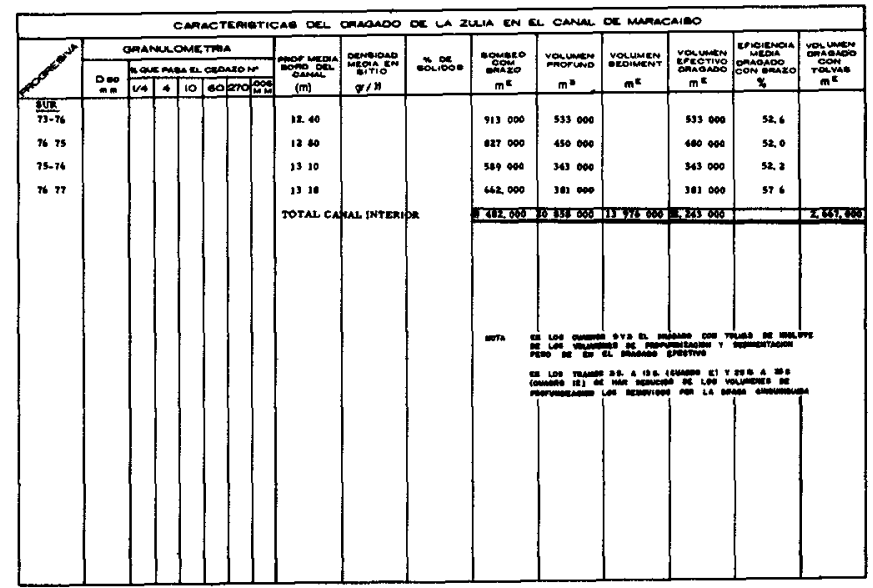




\section{"DRAGADO DE AGITACION CON BRAZO" EN EL CANAL DE MARACAIBO - VENEZUELA}

indicadas en las órdenes de dragado, lo cual no es rigurosamente cierto.

Sin embargo, las dificultades e imprecisiones anteriores desaparecen cuando se usan cifras globales y se obtienen a partir de ellas los va lores promedios de rendimiento y sedimentación. Con estas salvedades, se presentan separadamente para el Canal Interior y Exterior, las cifras de los cuadros siguientes:

\section{RELACION DE VOLUMENES DRAGADOS CON BRAZO \\ (Del 17-2-60 al 17-7-62)}

Descripción

Volumen de bombeo $\mathrm{m} 3$

Volumen de profun-

dización(incl. tolvas) $\mathrm{m} 3$

Volumen de sedimen

tación(incl. tolvas) $\mathrm{m} 3$

Volumen efectivo de

dragado

Eficiencia media del

dragado

m3 m3 32243000

49482000

20858000

13976000

$65.16 \%$
18962000

38219000

70462000

55181000

104663000

40249000

32938000

$67.32 \%$

\section{RELACION DE TIEMPOS}

TRABAJO EFECTIVO:

Tolvas:

Bombeo

Evoluciones

Ida y vuelta a descarga

Descarga

Total por Tolvas

Brazo:

Bombeo hacia el Este

Bombeo hacia el Oeste

Evoluciones

Total por Brazo

TRABAJO NO EFECTIVO:

Mantenimiento y reparaciones

Transferencias en el trabajo

Otras demoras

Mal tiempo

Tráfico

Cese

Accidentes

Total trabajo no efectivo

Tiempo total de operac.
Horas \% tiempo de operación

\begin{tabular}{rr}
1380.77 & 7.04 \\
160.35 & 0.82 \\
232.20 & 1.18 \\
64.98 & 0.33 \\
\hline 1838.30 & 9.37 \\
& \\
4466.22 & 22.78 \\
9686.08 & 49.40 \\
613.32 & 3.13 \\
\hline 14765.62 & 75.31
\end{tabular}

2738.23

13.97

9.28

0.05

1.95

0.01

2.98

0.02

12.10

0.06

85.57

0.44

151.47

3001.98

0.77

19605.90 


\section{COASTAL ENGINEERING}

A partir de las cifras dadas anteriormente, se obtienen los siguientes rendimientos medios:

a) Dragado con brazo:

Volumen bombeado por hora neta de bombeo $7088 \mathrm{~m} 3 /$ hora

Volumen efectivo dragado por hora neta de bombeo

4772

b) Dragado con tolvas:

Volumen dragado por hora de ciclo

$1524 \quad 11$

\section{REFERENCIAS}

The Hopper Dredge - Corps of Engineering - U.S. Army Government Printing Office - Washington - 1954.

Fundamentals of Soils Mechanics-Donald W. Taylor-John Wiley \& Sons New York - 1948.

Engineering Hydraulics -Hunter Rouse - John Wiley \& Sons -New York1958.

Paper on Sidecast (Boom) Dredging-Foreign Experience and Local appli cation at A.S. C. E. Fall Meeting by Henry G. A. Hayward-New York-1961. 